\title{
Communication simulation of on-board diagnosis network in high-speed Maglev trains
}

\author{
Zhigang LIU*, Yunchang HOU, Weijie FU \\ School of Electrical Engineering, Southwest Jiaotong University, Chengdu 610031, China
}

\begin{abstract}
The on-board diagnosis network is the nervous system of high-speed Maglev trains, connecting all controller, sensors, and corresponding devices to realize the information acquisition and control. In order to study the on-board diagnosis network's security and reliability, a simulation model for the on-board diagnosis network of high-speed Maglev trains with the optimal network engineering tool (OPNET) was built to analyze the network's performance, such as response error and bit error rate on the network load, throughput, and node-state response. The simulation model was verified with an actual on-board diagnosis network structure. The results show that the model results obtained are in good agreement with actual system performance and can be used to achieve actual communication network optimization and control algorithms.
\end{abstract}

Key words: Maglev trains; diagnosis network; OPNET; communication simulation

(C) 2011 JMT. All rights reserved.

\section{Introduction}

$\mathrm{T}$ he Maglev train is one of train systems with contactless levitation, guide, and drive. In terms of the magnetism between magnets and magnetic components on the track, the Maglev train is attracted to suspend above the track. The locomotion of trains depends on the traction of linear motors in vehicles [1-2].

During the travel of Maglev trains, efficient control and status messages involving train safety are transmitted and carried through a control network, and a great quantity of diagnosis messages about the electric and electronic devices are produced and transmitted through an on-board diagnosis network [3-4]. On-line diagnosis of all on board electric and electronic devices can be achieved with Maglev trains. Furthermore, some control functions not related to safety can be completed through the Maglev train on-board diagnosis network [5]. In high-speed Maglev trains, the main devices of on-board diagnosis network include a vehicle diagnosis computer, train diagnosis computer, and a train operation computer. In Maglev trains, a controller area network (CAN) with four CAN buses is composed of a vehicle diagnosis computer and all on-board electric and electronic devices. Each CAN bus contains about $30 \mathrm{CAN}$ nodes through which

Received Oct. 10, 2011; revision accepted Nov. 30, 2011

*Corresponding author. E-mail: liuzg_cd@126.com (Z.G. LIU)

(C) 2011 JMT. All rights reserved doi: 10.3969/j.issn.2095-087X.2011.04.004 all on-board electric and electronic devices can be connected together. In the train, the vehicle diagnosis computer, train diagnosis computer, and train operation computer compose an industrial Ethernet, which is used to connect all vehicles in the Maglev train [6-7]. In order to effectively design and develop the on-board diagnosis network, and optimize the diagnosis and control of Maglev trains [8], it is necessary to obtain the communication performance of the network.

In this paper, a simulation model of the on-board diagnosis network is built with the optimal network engineering tool (OPNET). It is used to analyze the network's performance, such as response error and bit error rate of the network load, throughput, and node-state response.

\section{On-board diagnosis network}

The on-board diagnosis network is a two-layer architecture network, including vehicle network (CAN bus) and train network (Ethernet). The vehicle network (CAN bus) is the core of the on-board diagnosis network. $\mathrm{CAN}$ is one of serial communication buses, which was developed by $\mathrm{BOSCH}$, and has become an international communication standard. Many features of CAN, such as message priority, multi-master work mode, flexible set, high reliability, and real time, make it applicable to train communications. Compared with train communication network (TCN), Lonworks and WordFIP, CAN has advantages of low price and flexibility [9]. According to ISO11898 [10], CAN includes a data link layer (DLL) 
and a physical layer. The DLL performs logical link control (LLC) and medium access control (MAC). The receiving and filtering functions, overload notice, and recover management of frames are completed in LLC. MAC is the core of CAN protocols, in which transport protocols such as control frame, arbitration, error check, and fault confinement are defined [11]. In Shanghai Maglev Line, CAN is adopted as the communication network of the on-board diagnosis system for highspeed Maglev trains [12].

There are many electric and electronic devices in Maglev trains, and it is necessary to divide the CAN into several parts, which will not only improve the efficiency of the CAN network but also enhance the security of the network. Thus, the fault of any bus part in the CAN network will not result in the collapse of the whole network. The devices to be diagnosed include all the electric and electronic devices in the vehicles. The diagnosis system consists of magnets levitation control unit, magnet guidance control unit, magnet brake control unit, linear generator converter, energy network distributor, energy network controller, vehicle control unit, door controller, location measurement unit, air conditioning controller, inverter, and fan unit. The number of devices to be diagnosed is over a hundred in each vehicle. The structure of a diagnosis system [5] is shown in Fig. 1.

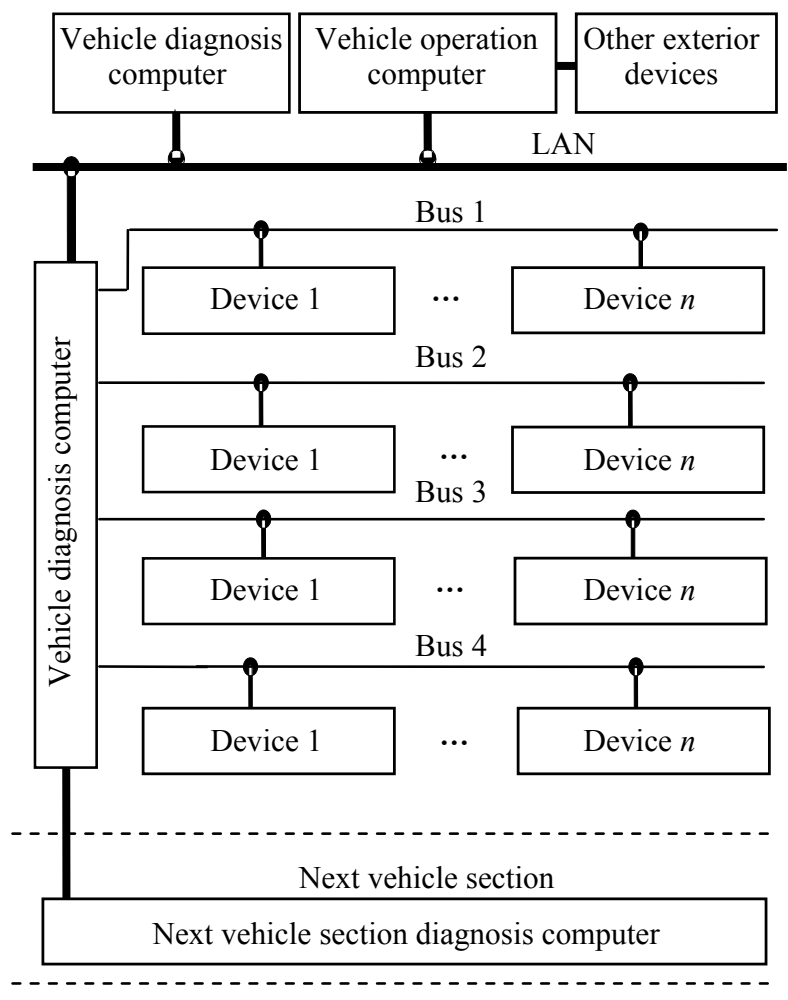

Ethernet

CAN
In the on-board diagnosis system, the vehicle diagnosis computer is the core unit, which is in charge of the entire diagnosis network in a vehicle. Their functions include sending network messages, such as period messages, control messages, and query messages, to $4 \mathrm{CAN}$ buses; receiving diagnosis messages from all on-board electric and electronic devices of 4 CAN buses; and, translating protocols between $\mathrm{CAN}$ and Ethernet.

\section{Modeling and simulation of diagnosis network}

\subsection{CAN node model}

Based on the actual structure of the diagnosis network, OPNET [13] is adopted to build a model of the whole on-board diagnosis network. The hierarchy modeling (network model, node model, and process model) [14] is used to build the on-board diagnosis network model. The network model is concerned with the network topology structure, including the distribution and number of nodes. The node model is composed of process models, which can constitute a complete protocol stack and reflect the features of actual devices. The process model is composed of finite state machines (FSM), by which the complete protocols can be described. The CAN node model in OPNET is built as shown in Fig. 2, which covers an application layer, a data link layer and a physical layer. The application layer includes input, output and "config" process modules, which are used to produce messages and response to messages. The functions of the application layer are realized through input and output modules, such as sending the data messages produced in application layer to the bus, or receiving data messages from the bus. The "config" process module is used to declare exterior files, by which the corresponding attributes of the whole network can be configured.

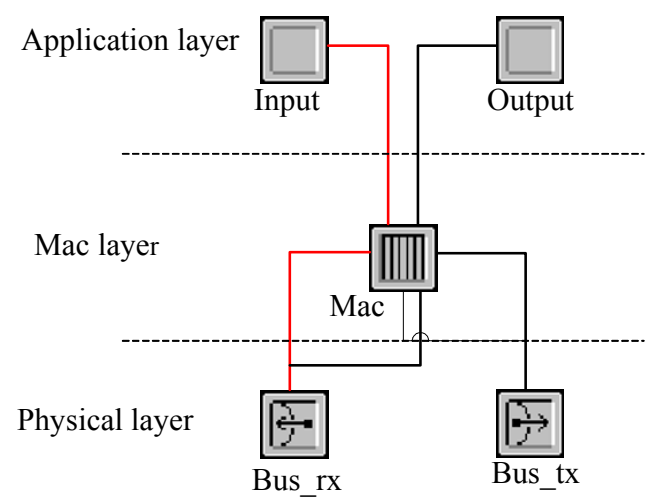

Fig. 2 CAN node model

Fig. 1 Diagnosis system structure 
MAC spearheads the functionality in a CAN protocol, since it processes the received messages from the bus and then sends messages from the application layer, such as responding message frame, control frame, arbitration, checking error, and error definition [15]. For the model in Fig. 3, firstly, the system is initiated to obtain parameters of the bus, and then assumes an idle state. In the idle state, the system can enter the sending or receiving state. The sending state means that the system can obtain data from the application layer, seal the data based on the data format of the CAN protocol, transmit the data to the physical layer for sending, and finally return to the idle state. The receiving state means that the system receives data from the bus. Once messages arrive in the bus, the receiving state is transited. After receiving data, arbitration state is transited. If new messages arrive in the next time interval, the system will return to the receiving state. In the arbitra- tion state, if no new messages arrive in a bit time, the system will directly enter into a decision state. The messages can be checked and processed in the decision state. If the received messages are errors or have no response, the system will enter into a receiving error state and sends an error frame. When the message is received successfully, the system will collect error statistics. If the error number is more than 128 , the system will enter into a sense only state, or else enter a waiting state. In the waiting state, if some messages are sent, the system will enter into the sending mode. If some messages are received, the system will enter into the idle mode. During 8-bit time waiting in the sense only mode, if some messages are received, the system will enter into the receiving state. If some messages are sent, the system will enter into the sending state. If no data is sent or received, the system directly enters into the idle state.

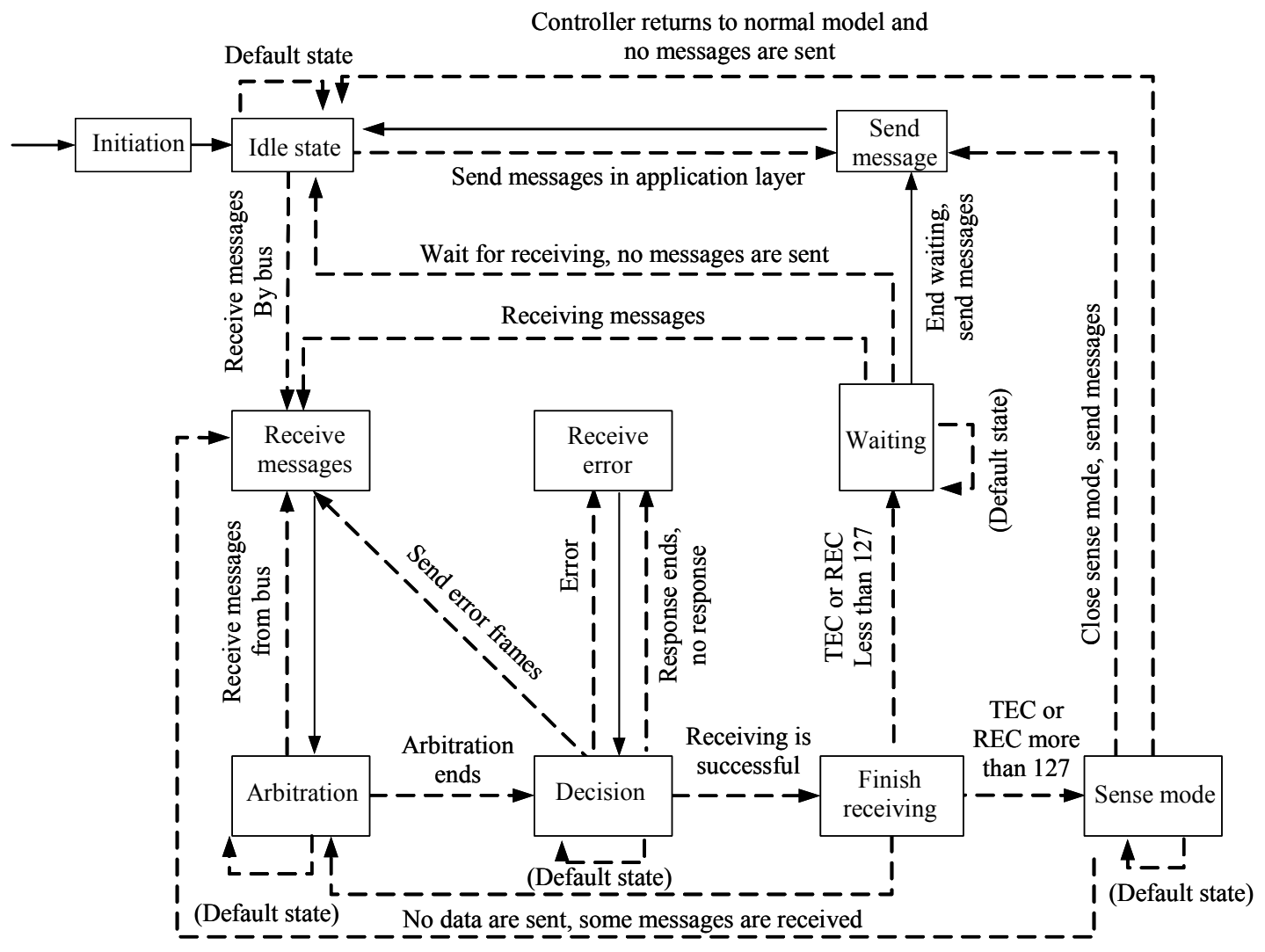

Fig. 3 The MAC structure of CAN node

\subsection{CAN network model}

The actual model of on-board diagnosis network constructed with OPNET for high-speed Maglev trains is shown in Fig. 4(a). In the model, the vehicle diagnosis computer includes four CAN buses as shown in Fig. 4(b). Each CAN node represents the actual corresponding electric or electronic device in Fig. 5. The ve- hicle diagnosis computer is the main node of the four CAN buses. The main node periodically sends the broadcast message in a standard frame to the four CAN buses, every 1-1.5 seconds. At the same time, the nonsafe control messages, related with air conditioner switch, headlight switch, and so on, will be sent in a standard frame to the four CAN buses. When each node of electric or electronic device receives the broadcast 
message, the status message frame of each node will be sent to the main node as the response. The correspond-

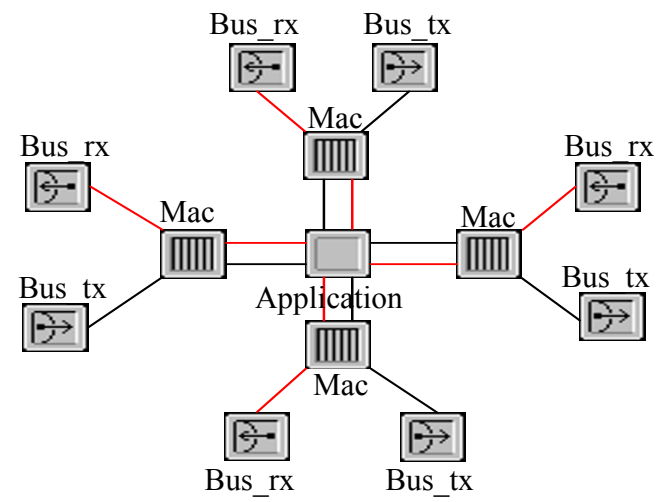

(a) Vehicle diagnosis computer model

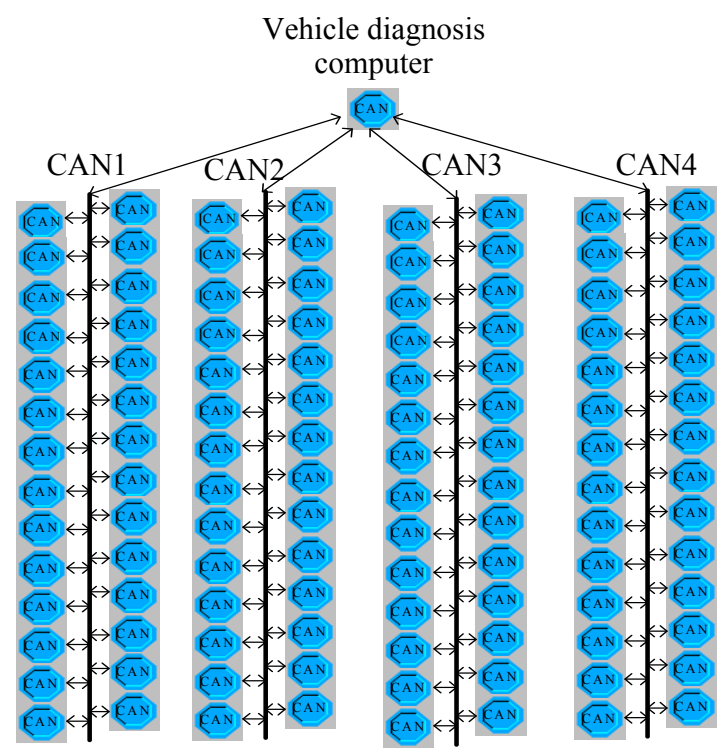

(b) The model of on-board diagnosis network of a highspeed Maglev train

Fig. 4 The models of whole network and main node with OPNET ing nodes associated with non-safe control can receive and process the control message based on the frame ID. If the main node cannot receive a given node's status message, the main node will send the single-node inquiry message to the node.

After three attempts, if the main node still cannot receive its status message, the node will be considered as a fault node. Because the control messages are periodically sent, the normal node associated with non-safe control can receive the control messages. According to the vehicle's length, the communication speed of $500 \mathrm{kbps}$ is adopted in the model. In a single CAN bus, the number and properties of each device node is listed in Table 1, where CAN1 is taken as an example. "uniform $(m)$ " and "exponential $(n)$ " mean the unvaried period and average period with exponential distribution, respectively.

Table 1 The number and properties of each device node

\begin{tabular}{ccc}
\hline Device node & Number & Data \\
\hline $\begin{array}{c}\text { Magnet levitation } \\
\text { control unit }\end{array}$ & 6 & uniform(0.02) \\
$\begin{array}{c}\text { Magnet guidance } \\
\text { control unit }\end{array}$ & 6 & uniform $(0.02)$ \\
$\begin{array}{c}\text { Magnet brake } \\
\text { control unit }\end{array}$ & 2 & uniform $(0.02)$ \\
$\begin{array}{c}\text { Door controller } \\
\text { Energy network } \\
\text { controller }\end{array}$ & 4 & uniform $(0.2)$ \\
$\begin{array}{c}\text { Passengers infor- } \\
\text { mation }\end{array}$ & 2 & $\begin{array}{c}\text { exponential(0.1), } \\
\text { uniform }(0.01) \\
\text { exponential }(1), \\
\text { uniform }(0.1)\end{array}$ \\
Fire alarm & 4 & $\begin{array}{c}\text { exponential(10), } \\
\text { uniform }(0.1)\end{array}$ \\
\hline
\end{tabular}

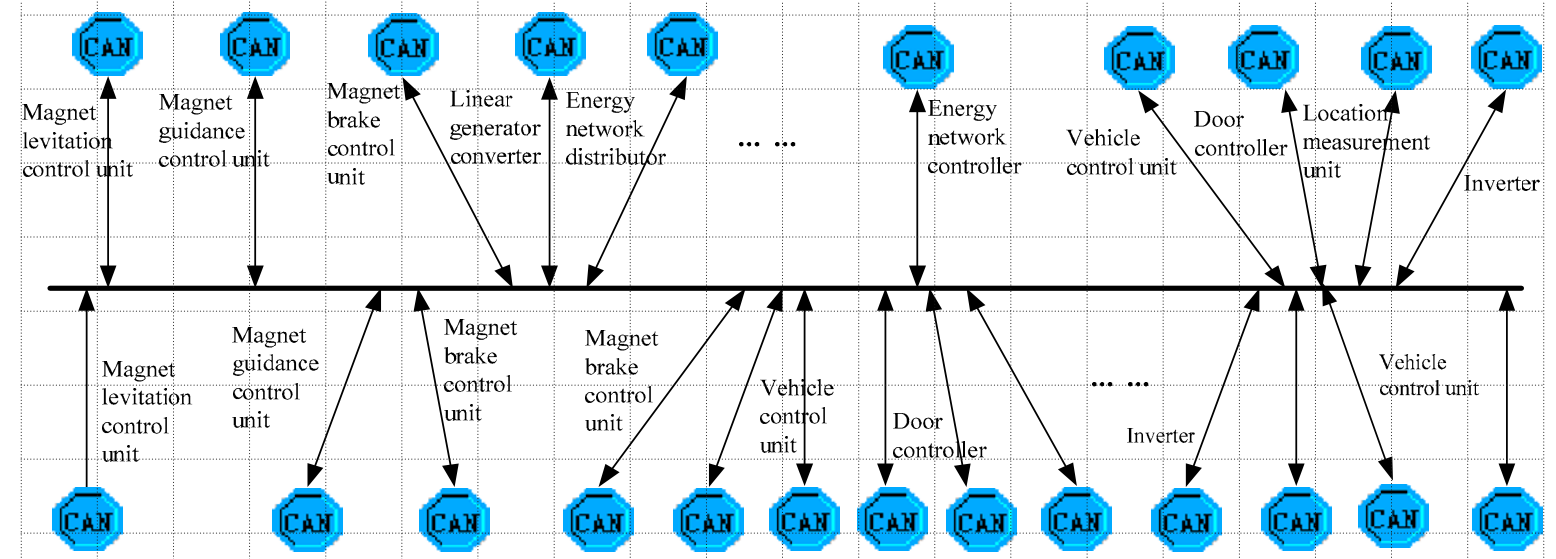

Fig. 5 The actual model of a single CAN bus with OPNET 


\section{Simulation results}

A Maglev train at a speed of $400 \mathrm{~km} / \mathrm{h}$ will cover $111.11 \mathrm{~m}$ per second. The on-board diagnosis system should have a high real-time performance and a high reliability. According to the normal network delay model, it includes the application layer delay, waiting delay, sending delay, transmission delay, and receiving delay. Because the application layer delay is related to hardware performance and actual applications, it is not considered in the simulation carried out. In addition, the transmission delay is related to communication length; hence, delays with short communication lengths can be ignored.

The standard of communication network QoS (quality of service) mainly includes network delay, bus load, response time, network throughput, etc. In this paper, the main factors that influence the on-board diagnosis network performance are investigated. The simulation analysis includes three parts as below:

(1) Analysis of the network performance for normal communication, such as the bus load and throughput for different error rates (BERs) and frame frequencies, and the relationship between average ETE (end to end) delay and priority for different bus loads and BERs.

(2) Analysis of the performance of on-board diagnosis network when changing the network topology. This includes changing the status of some electric and electronic device nodes, adding communication device node, and cutting communication device node from the network.

(3) Analysis of the network performance when changing the frequency of sending and receiving data packets for the device nodes. In order to obtain a high real-time performance, the time intervals of sending message for device nodes were reduced. We analyzed the network performance by changing the transfer characteristics of the device nodes.

The relationship between BER and bus load of the on-board diagnosis network is shown in Fig. 6, and the relationship between BER and bus throughput in Fig. 7 . We can find that the bus load of the on-board diagnosis network is about $11 \%$ and $15 \%$ for the BER of 0 and 0.0001 , respectively.

When BER increases to 0.001 (Fig. 6), the bus load of the on-board diagnosis network firstly increases sharply, then decreases with time, and remains constant at about $16 \%$ eventually. When $\mathrm{BER}=0.0001$, error frame and re-sending data make the number of frames on bus increase, and consequently, the load on the bus is increased further. When BER $=0.001$, based on the fault definition of CAN bus, some nodes are defined as fault nodes and log out, which makes the bus load decrease.

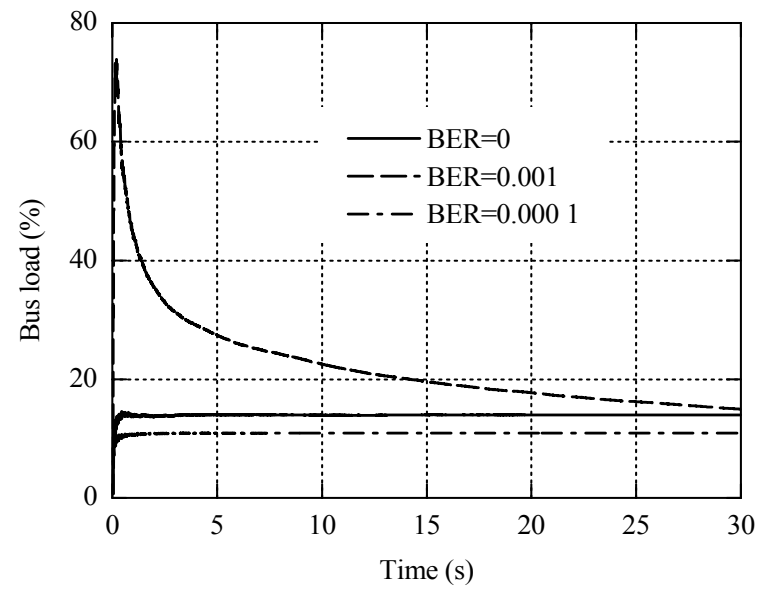

Fig. 6 Relationship between BER and bus load

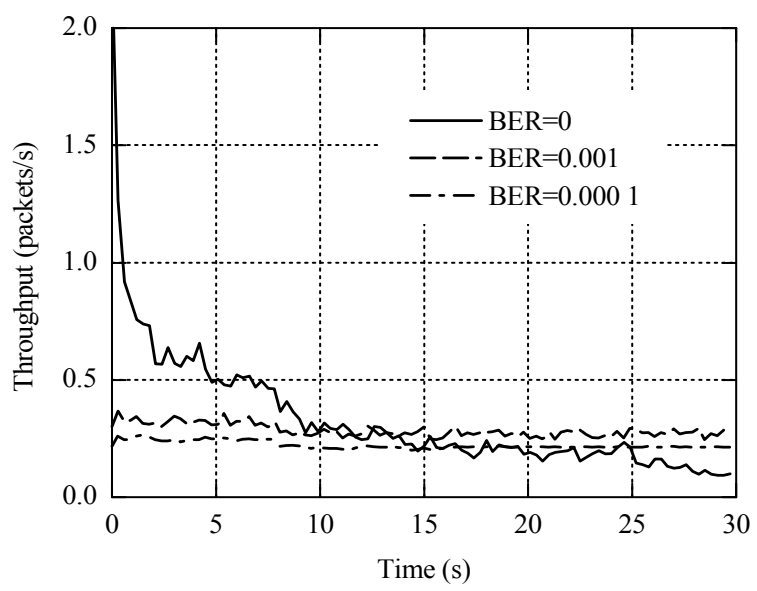

Fig. 7 Relationship between BER and throughput

A similar rule is observed for the relationship between BER and bus throughput.

Fig. 8 shows the relationship between frame frequency and bus load, and Fig. 9 the relationship between frame sending frequency and bus throughput. With the increase of frame sending frequency, the bus load and bus throughput increase with time accordingly. The relationship between average ETE delay and priority for different bus loads is shown in Fig. 10, and for different BERs in Fig. 11 . When the bus load is $8 \%$ and $12 \%$, the average ETE delay remains relatively constant at about 0.00018 . When the bus load is $31 \%$, however, the ETE delay increases in the priority range of 2 to 3 , and then remains relatively constant at about 0.00022 . The delay in the simulation depends on the queuing delay. In general, the low load ratio means less bus idle time and more waiting time of messages for idle bus. In Fig. 10, the queuing time decreases as the load variable ratio is reduced. When the load ratio increases, the delay intuitively increases. The analysis result is similar for the relation ship between 
BER and bus throughput. This is due to the fact that the high error rate makes the bus load increase, and some data frames re-send, which increases the queuing time.

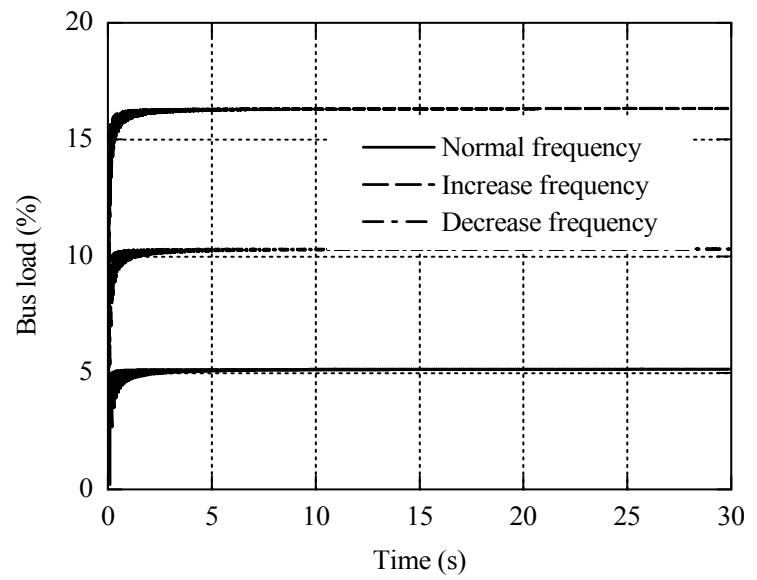

Fig. 8 Relationship between frame frequency and bus load

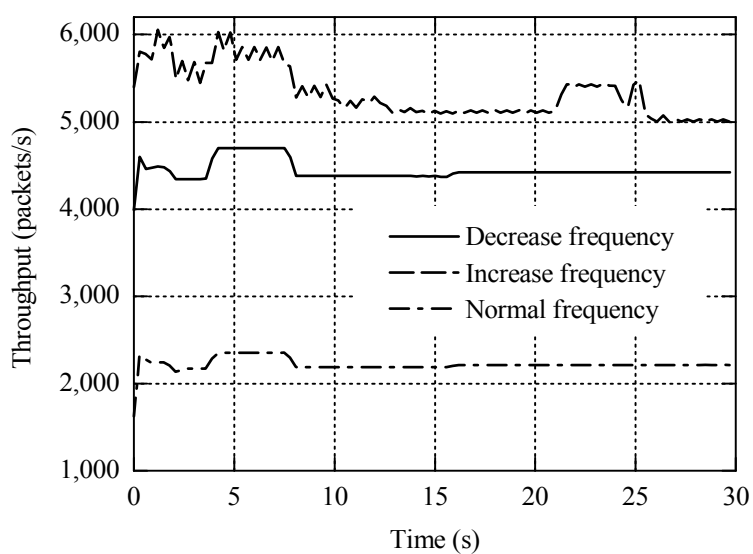

Fig. 9 Relationship between frame frequency and throughput

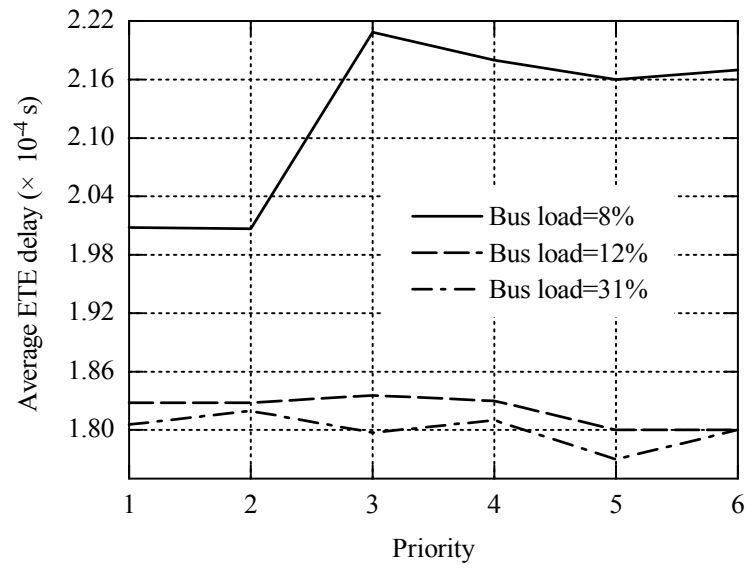

Fig. 10 ETE delay for different loads and different priorities

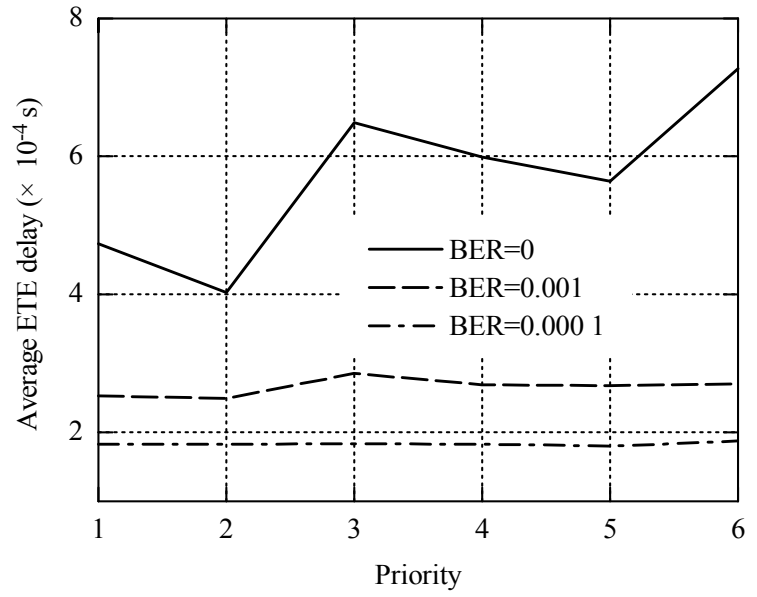

Fig. 11 ETE delay for different BERs and different priorities

\section{Conclusion}

In this paper, a simulation model of an on-board diagnosis network for high-speed Maglev trains with OPNET is built to analyze the network's performance. Based on the simulation results, BER is the most important influencing factor for the on-board diagnosis network performance, and the influence of response error on the network performance is small. Since the actual on-board diagnosis network of high-speed Maglev train is a complex system, the actual CAN node models are simplified for the simulation, which brings some limitations to the performance study of actual device nodes. The future work will focus on the node model improvement and parameters setting, which may provide a better support for the performance of actual on-board diagnosis network of high-speed Maglev train.

\section{Acknowledgements}

This study was supported by the National Natural Science Foundation of China (No. 51007074), the Program for New Century Excellent Talents in University (NECT-08-0825), the Research and Development Project of the National Railway Ministry (2011J016-B), and The basic research universities special fund operations (SWJTU11CX141).

\section{References}

[1] L.G. Yang, The maglev development and commercial application in China, IEEE Transaction on applied superconductivity, 2006, 16(2): 1138-1141.

[2] J.S. Wang, S.Y. Wang, J. Zheng, Recent development of high temperature superconducting Maglev system in China, IEEE Transaction on applied superconductivity, 2009, 19(3): 2142-2147. 
[3] H. Jiang, J. Lian, The development and review of Maglev Train in the world, Journal of the China Railway Society, 1991, 13(2): 88-93 (in Chinese).

[4] Z.G. Liu, Q. Wang, Y.D. Tan, The study of maglev train control and diagnosis networks based on role automation decentralization, IEICE Transaction on Information and System, 2008, 91(9): 2285-2292.

[5] Z.G. Liu, Q. Wang, Y.D. Tan, The study of maglev train control and diagnosis networks based on role automation decentralization, IEICE Transaction on Information and System, 2008, 91(9): 2285-2292.

[6] X. Wu, Maglev Train, Shanghai: Shanghai Science and Technology Press, 2003 (in Chinese).

[7] Z.Q. Long, S.S. Liu, C.K. Li, The research about communication network of Maglev train based on CAN bus, Electric Drive, 2001(2): 44-47 (in Chinese).

[8] D.V. Nuria, S. Alvaro, N. Carles, et al., Towards an optimized magnet-superconductor configuration in actual maglev devices, IEEE Transaction on applied superconductivity, 2011, 21(3): 1469-1472.

[9] X.H. Yang, Field Bus Technologies and Applications,
Beijing: Tsinghua University Press, 2003 (in Chinese).

[10] ISO 11898, Road vehicles-interchange of digital information-controller area Network for high speed communication ISO.

[11] W.J. Ni, A.J. Su, Q.Y. Guo, On-board information acquisition and diagnostic system for Maglev vehicle based on CAN-Bus, Electric Drive for Locomotives, 2006, 10(6): 25-28 (in Chinese).

[12] G. Hu, Z.G. Liu, G.F. Bo, Simulation of high-speed maglev on-board diagnosis system, Urban Mass Transit, 2011, 14(1): 52-55 (in Chinese).

[13] K. Salah, P. Calyamb, M.I. Buharic, Assessing readiness of IP networks to support desktop videoconferencing using OPNET, Journal of Network and Computer Applications, 2008, 31(4): 921-943.

[14] M. Chen, The Network Simulation of OPNET, Beijing: Tsinghua University Press, 2004 (in Chinese).

[15] J. Hao, J.C. Wu, C.Y. Guo, Modeling and simulation of CAN network based on OPNET, In: 2011 IEEE 3rd International Conference on Communication Software and Networks, Xi'an, 2011: 577-581.

(Editor: Junsi LAN) 